\title{
Analysis of Steady-State Common-Mode Response in Differential LC-VCOs
}

\author{
R. Doldán, A. J. Ginés, E. Peralías and A. Rueda \\ Instituto de Microelectrónica de Sevilla, CNM-CSIC, University of Seville, Spain \\ Emails: \{rdoldan, gines, peralias, rueda\}@imse-cnm.csic.es
}

\begin{abstract}
This paper analyzes the common-mode response of LC voltage-controlled oscillators (VCOs) in DC and periodic steady state regimes. The dependence of the common-mode voltage $\left(v_{\mathrm{cm}}\right)$ on the oscillation amplitude is theoretically studied. Closed and simple expressions for $v_{\mathrm{cm}}$ suitable for the VCO design and optimization are derived. The agreement with transistor level simulations has been verified in a $1.2 \mathrm{~V}$ lowpower 90nm CMOS case of study.
\end{abstract}

Key words: RF, LC-VCO, Common-Mode Voltage, VCO design and optimization.

\section{INTRODUCTION}

Integrated LC voltage-controlled oscillators (VCOs) are common functional blocks in modern radio frequency communication applications and one of the most critical sub-systems in the RF section. During the past decade, the VCO design towards low phase-noise and low-power consumption has been covered in several publications [1]-[5]. A deep theoretical comprehension of the oscillation mechanisms and source of degradations is achieved. This includes the non-linear nature of the amplitude self-maintained sustenance [2], analysis of phase-noise [3] and VCO transient response [4]-[5].

In the case of LC-VCOs, attention has been basically paid to the differential mode [1]-[5]. The impact of the commonmode at the output of the VCO $\left(v_{c m}\right)$ was not relevant since enough voltage margin was found in process nodes above $90 \mathrm{~nm}$. With technology scaling and trends towards lowvoltage designs, the situation has changed and some new effects should be considered in the design and optimization process [6]. Actually, for a particular design, $v_{c m}$ could suffer a voltage drop from 0 up to $100 \mathrm{mV}$ depending on the oscillation amplitude $(A)$. If this variation is not well controlled, the VCO performance could be degraded by extra phase noise (since more harmonics become relevant for noise folding-down) and it could become more sensitive to voltage supplies.

Additionally, any voltage variation should be considered in the circuit connected at the output of a VCO, since $v_{c m}$ defines its input common-mode. For instance, this is the typical case for I/Q phase generation using a DC-coupled divider-by-two (DIV2) [7]. Consequently, a good characterization of $v_{c m}$ is fundamental for the VCO-DIV2 optimization.

This work has been financed in part by the Junta de Andalucía project P09-TIC-5386 and the Ministerio de Economía y Competitividad project TEC2011-28302, both of them co-financed by the FEDER program. .
In this work a theoretical study of the common-mode voltage is performed. The dependence of $v_{c m}$ on $A$ is analytically justified. Contents in the paper are distributed as follows. In Sect. II, the proposed LC-VCO model and followed notation are introduced. Section III analyzes the non-linear behavior of the VCO active section. A first order transistor model is considered to provide closed-form intuitive relationships. Both common and differential modes are covered for completeness. In Sect. IV, a real VCO design in 90nm with active DIV2 load is presented for validation. Finally, conclusions are drawn.

\section{PROPOSED MODEL FOR LC-VCO ANALYSIS}

Fig.1a shows a simplified schematic of a well-known CMOS LC-VCO. A case of study with complementary crosscoupled active section and PMOS current source $\left(I_{B}\right)$ is considered for illustration purpose (results herein presented can be similarly derived for other VCO topologies). An equivalent model of the circuit, which describes both differential and common-mode, is depicted in Fig. 1b. The proposed model is constituted by a memory-less active block [8] connected to a lossy resonator tank $\left(L_{p}, C_{p}, R_{p}\right)$. This model implicitly assumes three basic hypotheses. These are: a) non quasi-static effects are negligible [9], and hence, the oscillation frequency $\omega_{0}$ is well below the transistor $f_{T}$, b) the circuit has a differential topology symmetry, and therefore, the currents through terminals $v_{1}$ and $v_{2}$ are balanced, $I\left(v_{1}\right)=-I\left(v_{2}\right)=i_{\mathrm{d}}$, and the tank common-mode current is negligible, and c) parasitics due to transistors and passive devices can be grouped on the tank elements, being their values,

$$
\begin{aligned}
& L_{p}=L \quad ; \quad C_{p}=C+C_{\text {load }}+1 / 2\left(C_{M O S, P}+C_{M O S, N}\right) \\
& R_{p}=\left(G_{p L}+G_{p C}+g_{d s p} / 2+g_{d s n} / 2\right)^{-1} \\
& C_{M O S}=4 C_{g d}+C_{g s}+C_{g b}+C_{d b}
\end{aligned}
$$

where $G_{p L}$ and $G_{p C}$ are the parasitic parallel conductance of tank-inductor and tank-capacitor respectively. The rest of parameters correspond to MOS transistors.

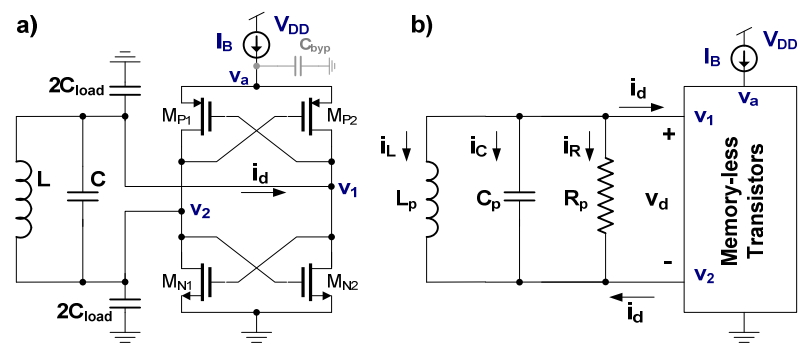

Figure 1. a) Simplified LC-VCO schematic; b) proposed model. 


\section{A. General VCO Description}

The memory-less active block in Fig. $1 \mathrm{~b}$ is in practice a four-terminal device. Bias $v_{\mathrm{a}}$ and ground nets must be explicitly included to describe the common-mode behavior. The response of this block will be generally given by a set of functions of the current and voltages at the different nets,

$$
\boldsymbol{f}\left(i_{d}, I_{B}, v_{1}, v_{2}, v_{a}\right)=0
$$

This set of functions is suitable for all instants in the VCO evolution, since the active-block does not have memory elements. In addition to this constrain, the circuit model in Fig.1b must satisfy the Kirchhoff's current law (KCL) for charge conservation, and hence,

$$
i_{d}+i_{L}+i_{C}+v_{d} / R_{p}=0 ; i_{C}=C_{p} \frac{d v_{d}}{d t} ; v_{d}=L_{p} \frac{d i_{L}}{d t}
$$

Taking the derivative of the first term in (3) and substituting the inductor and capacitor laws, we arrive at,

$$
\frac{d^{2} v_{d}}{d t^{2}}+\frac{1}{R_{p} C_{p}} \frac{d v_{d}}{d t}+\frac{1}{L_{p} C_{p}} v_{d}+\frac{1}{C_{p}} \frac{d i_{d}}{d t}=0
$$

Given the initial capacitor and inductor conditions, the VCO dynamic response is fully characterized by (2), (4), and the definition $v_{d}=v_{1}-v_{2}$. This implies solving an ordinary differential equation (ODE) joint with a non-differential non-linear equation set.

\section{B. Particulatization for Differential VCO Topologies}

For differential VCO topologies, such as the example of Fig.1a, the previous general analysis can be drastically simplified. Actually, due to the active section symmetry, we propose to express the constitutive relationships in (2) as an uncoupled set of the following three functions,

$$
\begin{aligned}
& \boldsymbol{f}\left(i_{d}, I_{B}, v_{1}, v_{2}, v_{a}\right)=0 \Leftrightarrow \\
& \left\{\begin{array}{c}
i_{d}=f_{d}\left(v_{d}, I_{B}\right) \\
v_{c m}=f_{c m}\left(v_{d}, I_{B}\right) \\
v_{a}=f_{a}\left(v_{c m}\right)
\end{array}\right\}, \quad \begin{array}{l}
v_{1}=v_{c m}+v_{d} / 2 \\
v_{2}=v_{c m}-v_{d} / 2
\end{array}
\end{aligned}
$$

The details of the uncoupling method will be developed in Sect. III. Appling this equivalence to (4), it follows,

$$
\frac{d^{2} v_{d}}{d t^{2}}+\frac{1}{C_{p}}\left(\frac{1}{R_{p}}+\frac{\partial f_{d}}{\partial v_{d}}\right) \frac{d v_{d}}{d t}+\omega_{0}^{2} v_{d}=0 ; \omega_{0}=\frac{1}{\sqrt{L_{p} C_{p}}}
$$

which is now a non-linear ODE in $v_{d}$ that can be solved independently. Having its solution, the other variables are directly obtained by substitution in (5).

Traditional studies have exclusively focused on the differential part [1]-[5] and do not worry about the common-mode dependence [6]. For practical VCO designs verifying the startup condition [8], $1 / R_{\mathrm{p}}<-\left.\left(d f_{d} / d v_{d}\right)\right|_{v \mathrm{~d}=0}$, the stationary solution of (6) will be a periodical signal, generally given by,

$$
v_{d}(t) \underset{t \rightarrow \infty}{=} \sum_{n \geq 1} A_{n} \cos \left(n \omega_{o} t+\phi_{n}\right)
$$

where $A_{\mathrm{n}}$ and $\phi_{\mathrm{n}}$ are the amplitude and phase parameters for the different Fourier's series terms. If the quality factor of the tank is much higher than one as usual, $Q=\omega_{0} R_{\mathrm{p}} C_{\mathrm{p}}>>1$, a nearly sinusoidal is found in the stationary situation,

$$
v_{d}(t) \underset{t \rightarrow \infty}{\approx} A \cos \left(\omega_{o} t+\phi\right)
$$

In [4]-[5], closed-form equations for the amplitude $A$ and oscillation frequency $\omega_{0}$ using level-1 transistor models are derived. These relationships provide a very interesting information and intuitive view for the VCO design. In this work, we apply the same formalism to the analysis of the commonmode, that is, to obtain the curve $v_{c m}=f_{c m}\left(v_{d}, I_{\mathrm{B}}\right)$. For completeness, the expressions of $v_{d}=f_{d}\left(v_{d}, I_{\mathrm{B}}\right)$ and $v_{\mathrm{a}}=f_{\mathrm{a}}\left(v_{\mathrm{cm}}\right)$ will be also derived.

\section{Common-Mode Analysis}

Under our proposal, the function $v_{c m}=f_{c m}\left(v_{d}, I_{\mathrm{B}}\right)$ describes the common-mode response $v_{c m}(t)$ at any time. In practice, only the stationary time average of this wave, $V_{\mathrm{cm}} \mathrm{sT}$, is relevant, since current source $I_{B}$ is usually decoupled from the active block, at any frequency, for correct VCO operation $\left(\mathrm{C}_{\text {byp }}\right.$ in Fig. 1a). It is worth noticing that the $v_{\mathrm{cm}}$ voltage at quiescent state $\left(v_{\mathrm{cm}}\right.$ at $\left.v_{d}=0\right)$ could significantly vary from the value at steady state $V_{\mathrm{cm}}{ }^{\mathrm{ST}}$ [6]. Actually, up to $100 \mathrm{mV}$ difference respect to the expected quiescent point value could be found in current CMOS $90 \mathrm{~nm}$ process, and therefore, the existing drop should be considered during design. This phenomenon can be theoretically evaluated using the function $v_{c m}=f_{c m}\left(v_{d}, I_{\mathrm{B}}\right)$ under the oscillation voltage in (7). The output signal will be also periodic, but in this case with a half period due to even symmetry of $f_{c m}$ function (see Sect. III). The resulting value in steady state condition, $V_{\mathrm{cm}} \mathrm{ST}$, is given by the Fourier's series coefficient of order zero as,

$$
V_{c m}^{S T}=\left\langle v_{c m}(t)\right\rangle=\frac{\omega_{0}}{\pi} \int_{t_{o}}^{t_{o}+\pi / \omega_{0}} f_{c m}\left(v_{d}(t)\right) d t
$$

which using (8) for nearly sinusoidal VCOs simplifies to,

$$
V_{c m}^{S T}\left(A, I_{B}\right)=\frac{1}{\pi} \int_{0}^{\pi} f_{c m}\left(A \sin \theta, I_{B}\right) d \theta=\frac{2}{\pi} \int_{0}^{A} \frac{f_{c m}\left(x, I_{B}\right)}{\sqrt{A^{2}-x^{2}}} d x
$$

explicitly showing the dependence of the common-mode on the oscillation amplitude $A$.

\section{MEMORY-LESS ACTIVE BLOCK DESCRIPTION}

We have stated before that the memory-less active block is completely characterized by the non-linear functions $f_{\mathrm{d}}, f_{\mathrm{cm}}$ and $f_{\mathrm{a}}$. Obtaining this information requires, in general, the use of electrical simulations and numerical methods due to the complexity of the compact $i-v$ relations of MOS transistors. Since $f_{\mathrm{d}}, f_{\mathrm{cm}}$ and $f_{\mathrm{a}}$ are functions of a memory-less block, we can get the relationships between voltages and currents in static behavior, and then extend them for any regime. Test set-up for dc-sweep simulations consist of exciting the active block with a dc current source, $i_{d}$, between the block input terminals $v_{1}$ and $v_{2}$. Then, voltages at nodes $v_{1}, v_{2}$ (or $v_{d}, v_{c m}$ ) and $v_{\mathrm{a}}$ are taken for each $i_{d}$ value considering bias current $I_{B}$ as a fixed parameter. Finally, results are expressed according to (5) following the next general procedure. 


\section{A. General Derivation of the Active Block Functions}

The derivation of the memory-less block constitutive function requires an analysis of the transistor current relationships,

$$
I_{B} \stackrel{(1)}{=} i_{p 1}+i_{p 2} \stackrel{(2)}{=} i_{n 1}+i_{n 2} \quad ; \quad i_{d} \stackrel{(3)}{=} i_{n 2}-i_{p 2} \stackrel{(4)}{=} i_{p 1}-i_{n 1}
$$

where $i_{p k}$ is the drain output current of PMOS $\mathrm{M}_{\mathrm{Pk}}$, and $i_{n k}$ is the drain input current of NMOS $\mathrm{M}_{\mathrm{Nk}}$ in Fig. 1a. Taking into account the dependence of the MOS drain current on its terminal voltages, we can proceed as follows to get the wanted expressions. Considering the equality (2) in (11),

$$
\Phi_{c}\left(v_{1}, v_{2}, I_{B}\right)=I_{B}-i_{n 1}\left(v_{1}, v_{2}\right)-i_{n 2}\left(v_{2}, v_{1}\right)=0
$$

and substituting, $v_{1}=v_{\mathrm{cm}}+v_{\mathrm{d}} / 2, v_{2}=v_{\mathrm{cm}}-v_{\mathrm{d}} / 2$, the commonmode function $f_{\mathrm{cm}}$ is obtained by working out $v_{c m}$ as,

$$
\Phi_{c}\left(v_{c m}, v_{d}, I_{B}\right)=0 \Rightarrow v_{c m}=f_{c m}\left(v_{d}, I_{B}\right)
$$

The topological symmetry in this part of the active block implies that a change of sign in $v_{d}$ will not change the value of $v_{c m}$, and therefore $f_{c m}$ must be an even function of $v_{d}$. Applying the same substitutions in the equalities (3)-(4) in (11), we arrive at,

$$
\begin{gathered}
\Phi_{a}=i_{p 1}\left(v_{a}, v_{1}, v_{2}\right)-i_{n 1}\left(v_{1}, v_{2}\right)+i_{p 2}\left(v_{a}, v_{2}, v_{1}\right)-i_{n 2}\left(v_{2}, v_{1}\right)=0 \\
\Downarrow \\
\Phi_{a}\left(v_{a}, v_{c m}, v_{d}\right)=0 \Rightarrow v_{a}=f_{a}\left(v_{c m}\right)
\end{gathered}
$$

where the inverse of $f_{c m}, v_{d}=f_{c m}^{-1}\left(v_{c m}, I_{B}\right)$, is applied to get the relationship between $v_{a}$ and $v_{c m}$. To conclude, the dependence of $i_{\mathrm{d}}$ on the differential voltage is derived from equality (4) in (11) considering the previous results, in the form,

$$
i_{d}=i_{p 1}\left(v_{a}, v_{1}, v_{2}\right)-i_{n 1}\left(v_{1}, v_{2}\right) \Rightarrow i_{d}=f_{d}\left(v_{d}, I_{B}\right)
$$

\section{B. Closed-form Derivation using Level-1 Transistor Model}

Solving the previous set of operations for MOS advanced level models could be a hard task and surely approximations by numerical methods have to be applied. Nevertheless, simple and closed forms can be derived when a level-1 MOS model is considered. As demonstrated below, this approach is good enough to describe an actual LC-VCO implementation whichever advanced level model it employs.

Let us assume, for further simplification and compactness of expressions, a common absolute value of the threshold voltages, equal effective transconductance, and no channel length modulation for NMOS and PMOS transistors,

$$
V_{T}=V_{T, N}=-V_{T, P} ; \beta=k_{N}^{\prime} \frac{W_{N}}{L_{N}}=k_{P}^{\prime} \frac{W_{P}}{L_{P}} ; \lambda_{N}=\lambda_{P}=0
$$

After analyzing all possible cases of operation regions for transistors in Fig. 1a, the complete behavior of the VCO can be summarized as a function of the differential voltage $v_{d}$ excursion, and classified according to the parameter $V_{\mathrm{B}}=\sqrt{ }\left(2 \mathrm{I}_{\mathrm{B}} / \beta\right)$. That is presented in Table I, where results for $v_{\mathrm{d}}<0$ are omitted due to their even symmetry. Two different situations depending on the parameter $V_{\mathrm{B}}$ are distinguished, being the case $V_{B}<V_{T}$ the most interesting in low-voltage low-power applications [10]. We focus on this situation.
Any couple of transistors diagonally opposite, $\left(\mathrm{M}_{\mathrm{P} 1}, \mathrm{M}_{\mathrm{N} 2}\right)$ and $\left(\mathrm{M}_{\mathrm{P} 2}, \mathrm{M}_{\mathrm{N} 1}\right)$ are always in the same operation region. This relationship is justified because gate and drain to source voltages, $\left|v_{\mathrm{GS}}\right|,\left|v_{\mathrm{DS}}\right|$, are always equal in these transistors. Attending to the voltage $v_{\mathrm{d}}$ value, three cases of study can be distinguished. In Case $1, v_{d}$ is close to 0 below $V_{B}$ and all transistors remain in saturation. VCO quiescent state belongs to this case. When increasing $v_{d}$ up to $V_{T}$, the circuit enters in the Case 2. In this situation, $\left(\mathrm{M}_{\mathrm{P} 2}, \mathrm{M}_{\mathrm{N} 1}\right)$ maintains its operation, and $\left(\mathrm{M}_{\mathrm{P} 1}, \mathrm{M}_{\mathrm{N} 2}\right)$ pass to cut-off region, since their voltage gates $\left(v_{1}, v_{2}\right)$ increase and decrease, respectively. Finally, in Case 3 , with $v_{d}$ above $V_{T}$, the couple $\left(\mathrm{M}_{\mathrm{P} 2}, \mathrm{M}_{\mathrm{N} 1}\right)$ enters in ohmic region while $\left(\mathrm{M}_{\mathrm{P} 1}, \mathrm{M}_{\mathrm{N} 2}\right)$ continues in cut-off.

TABLE I. OPERATION REGIONS FOR TRANSISTORS IN ACTIVE BLOCK

\begin{tabular}{||c||c|c||c|c|}
\cline { 2 - 5 } \multicolumn{1}{c|}{} & \multicolumn{2}{c||}{$V_{B}<V_{\mathrm{T}}$} & \multicolumn{2}{c|}{$V_{B}>V_{\mathrm{T}}$} \\
\cline { 2 - 5 } \multicolumn{1}{c|}{ Case } & $\mathrm{M}_{\mathrm{P} 2}, \mathrm{M}_{\mathrm{N} 1}$ & $\mathrm{M}_{\mathrm{P} 1}, \mathrm{M}_{\mathrm{N} 2}$ & $\mathrm{M}_{\mathrm{P} 2}, \mathrm{M}_{\mathrm{N} 1}$ & $\mathrm{M}_{\mathrm{P} 1}, \mathrm{M}_{\mathrm{N} 2}$ \\
\hline 1: $0<v_{d}<V_{B}$ & saturation & saturation & saturation & saturation \\
\hline 2: $V_{B}<v_{d}<V_{T}$ & saturation & cut-off & ohmic & saturation \\
\hline 3: $V_{T}<v_{d}<V_{D D}$ & ohmic & cut-off & ohmic & cut-off \\
\hline
\end{tabular}

After this qualitative analysis, a mathematical description of the different operation conditions could be performed. Let us show the analysis of the Case 1 with $V_{B}<V_{T}$ as an example. In this case, if $0 \leq\left|v_{d}\right| \leq V_{B}$, all the transistors are in saturation. Considering a level-1 transistor model for this region in the procedure of Sect. III.A, we arrive at,

$$
\begin{aligned}
& V_{B}^{2}=\left(v_{1}-V_{T}\right)^{2}+\left(v_{2}-V_{T}\right)^{2} \Rightarrow v_{c m}=V_{T}+\sqrt{2 V_{B}^{2}-v_{d}^{2}} / 2 \\
& \left(v_{1}-V_{T}\right)^{2}+\left(v_{2}-V_{T}\right)^{2}=\left(v_{a}-v_{1}-V_{T}\right)^{2}+\left(v_{a}-v_{2}-V_{T}\right)^{2} \Rightarrow v_{a}=2 v_{c m} \\
& i_{d}=\frac{\beta}{2}\left(\left(v_{a}-v_{1}-V_{T}\right)^{2}-\left(v_{1}-V_{T}\right)^{2}\right)=-\frac{\beta}{2} v_{d} \sqrt{2 V_{B}^{2}-v_{d}^{2}}
\end{aligned}
$$

Using a similar handling for the rest of cases (omitted for space limitation), the constitutive functions of the active block with $V_{\mathrm{B}}=\sqrt{ }\left(2 \mathrm{I}_{\mathrm{B}} / \beta\right)$ are finally derived as:

$$
\begin{aligned}
& \text { - } v_{c m}=f_{c m}\left(v_{d}, I_{B}\right)= \\
& \begin{cases}V_{T}+\sqrt{2 V_{B}^{2}-v_{d}^{2}} / 2, & \text { if } 0 \leq\left|v_{d}\right| \leq V_{B} \\
V_{T}+V_{B}-\left|v_{d}\right| / 2, & \text { if } V_{B} \leq\left|v_{d}\right| \leq V_{T} \\
V_{T}-\left|v_{d}\right| / 2+\sqrt{\left(\left|v_{d}\right|-V_{T}\right)^{2}+V_{B}^{2}}, & \text { if } V_{T} \leq\left|v_{d}\right| \leq V_{D D}\end{cases} \\
& \text { - } i_{d}=f_{d}\left(v_{d}, I_{B}\right)= \begin{cases}-\beta v_{d} \sqrt{2 V_{B}^{2}-v_{d}^{2}} / 2, & \text { if } 0 \leq\left|v_{d}\right| \leq V_{B} \\
-I_{B} \operatorname{sign}\left(v_{d}\right), & \text { if } V_{B} \leq\left|v_{d}\right| \leq V_{D D}\end{cases} \\
& \text { - } v_{a}=f_{a}\left(v_{c m}\right)=2 v_{c m}, \quad \forall\left|v_{d}\right| \leq V_{D D}
\end{aligned}
$$

In order to validate the theoretical predictions using (10) and (18), a VerilogA transistor level-1 model was implemented under Cadence FrameWork II. Figure 2 shows the comparison with Spectre RF results for both the DC and transient steady state simulations of a LC-VCO implementation. The value of $v_{c m}$ versus the differential voltage $v_{d}$ and the $\mathrm{V}_{\mathrm{cm}}$ ST dependence on the oscillation amplitude $A$ are depicted, respectively. This implementation uses $V_{\mathrm{DD}}=1.2 \mathrm{~V}$, $I_{\mathrm{B}}=320 \mu \mathrm{A}, V_{\mathrm{T}}=0.38 \mathrm{~V}$ and $\beta=65.17 \mathrm{~mA} / \mathrm{V}^{2}, L=1 \mathrm{nH}, C=1 \mathrm{pF}$ and $C_{\text {load }}=0 \mathrm{~F}$. The oscillation amplitude has been controlled assuming different parasitic parallel resistances of the tank, $R_{p} \in[0.22,2.95] \mathrm{k} \Omega$. The discrepancy between theoretical and 


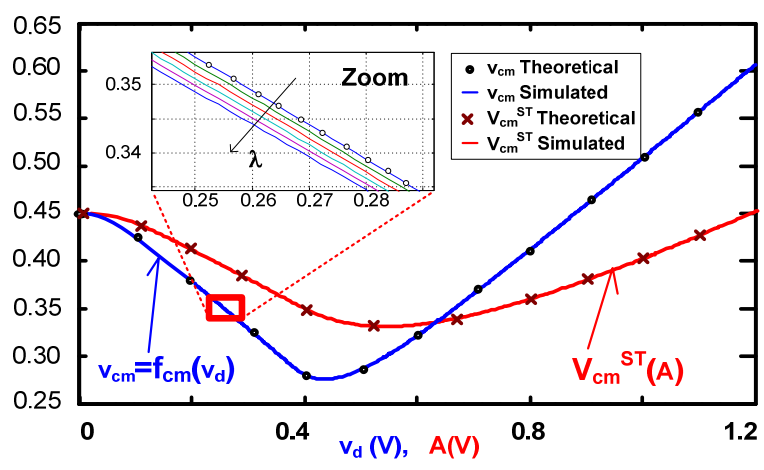

Figure 2. Comparison between theoretical preditions and Spectre RF results using a VerilogA model $\left(\lambda=0\right.$ to $0.5 \mathrm{~V}^{-1}$ in steps of $\left.0.1 \mathrm{~V}^{-1}\right)$.

simulation results is almost negligible even if channel length modulation is considered, $\lambda \in[0,0.5] \mathrm{V}^{-1}$.

\section{AN EXAMPLE IN A RF 90NM CMOS PROCESS}

In this section, the theoretical analysis of the commonmode is validated in a real VCO design in a RF 90nm CMOS process. The VCO, depicted in Fig.3, uses a $1 \mathrm{nH}$ differential inductor and MIM capacitor array with $1 \mathrm{pF}$ total capacitance. A DIV2 based on two source-coupled latches (SCL) is considered as active load. Figure 4 shows the transient evolution of the VCO common-mode and the corresponding oscillation voltage. As expected from (18), $v_{\mathrm{cm}}$ varies from the initial quiescent state as a function of the oscillation amplitude $A$. This behavior could degrade the performance of the VCO due to the additional phase noise, but it becomes critical for the DIV2 operation, since $v_{\mathrm{cm}}$ defines its input level in a DCcoupled scheme. The effect has been traditionally overcome by extra design margin, since VCO-DIV2 co-simulation was only performed at the final design steps. Early co-simulation with Spectre RF results is not time-effective. Actually, more than $1 / 2$ hour (Intel Core II $2.6 \mathrm{GHz}$ CPU) is need for a single iteration on the VCO design in nominal conditions.

In our work this simulation time has been reduced in several orders of magnitude without a significant accuracy penalty, and hence, making VCO-DIV2 co-design feasible early in the design phase. Figure 5 repeats the simulation in Fig. 2 and it includes: a) the characteristic of the common-mode $f_{c m}$ using a simple DC-sweep simulation, b) the dynamic response evaluated in SpectreRF from 50 periodic steady state analysis, c) our theoretical prediction using (10) over the curve a). The computation time for the theoretical curve is below 1s. Notice that the error between b) and c) is in the worst case below $18 \mathrm{mV}$.

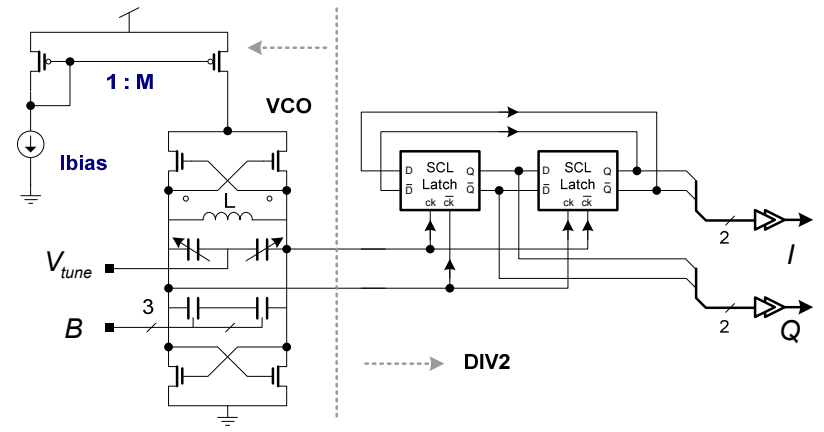

Figure 3. Simplified schematic of the LC-VCO with DIV2 load.

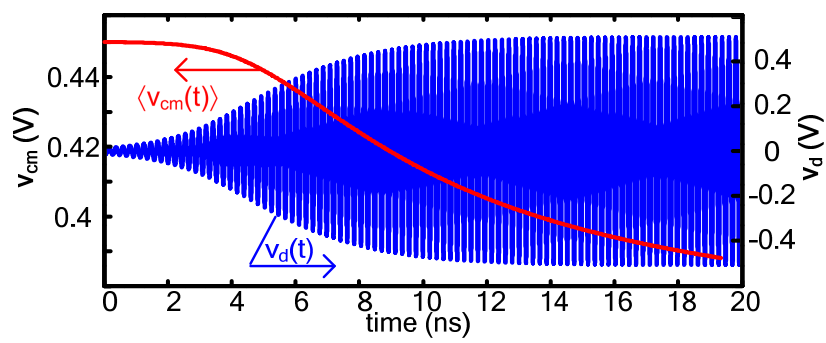

Figure 4. Transient evolution of $v_{\mathrm{cm}}$ showing the dependece on $v_{\mathrm{d}}$.

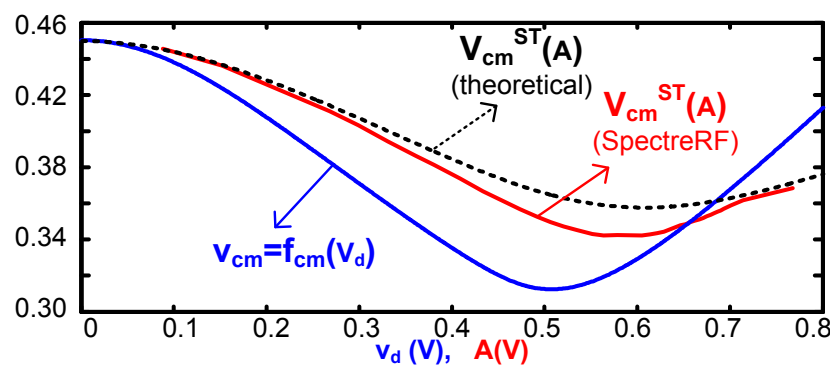

Figure 5. Comparison between theoretical preditions and Spectre RF results for the $90 \mathrm{~nm}$ CMOS case of study.

This discrepancy is justified by the drastic topological reduction of the circuit in Fig. 1a respect to the model in Fig. 1b.

\section{CONCLUSIONS}

A theoretical study of the stationary common-mode in LC VCOs is done in this work. The dependence of the commonmode voltage, $v_{\mathrm{cm}}$, on the oscillation amplitude is theoretically studied. Closed and simple expressions for $v_{\mathrm{cm}}$ suitable for the VCO design and optimization are derived. They provide an accurate prediction of the $v_{\mathrm{cm}}$ drops which minimizes risk in current low-voltage low-power applications at an early design phase due to the reduction of voltage room.

\section{REFERENCES}

[1] D. Ham and A. Hajimiri , "Concepts and methods in optimization of integrated LC VCOs," IEEE Journal of Solid-State Circuits, vol.36, no.6, pp.896-909, June 2001.

[2] Hegazi, J. Rael and A. Abidi, The Designer's guide of High Purity Oscillators, Kluwer, 2005.

[3] A. Demir, et al., "Phase noise in oscillators: a unifying theory and numerical methods for characterization," IEEE Trans. Cir. and Sys. I, vol.47, no.5, pp.655-674, May 2000.

[4] A. Buonomo, "Nonlinear Analysis of Voltage-Controlled Oscillators: A Systematic Approach," IEEE Trans. Cir. and Sys. I, vol.55, no.6, pp.1659-1670, July 2008 .

[5] A. Buonomo and A. Lo Schiavo, "Determining the oscillation of differential VCOs," IEEE Circuits and Systems, ISCAS, vol.3, pp. 144147, May 2003

[6] P. Vanassde, G. Gielen and W. Sansen, Systematic Modeling and Analysis of Telecom Frontends and their Building Blocks, Springer 2005.

[7] A. Ginés. et al. , "On-chip biased voltage-controlled oscillator with temperature compensation of the oscillation amplitude for robust I/Q generation," IEEE Circuits and Systems, ISCAS, pp.1979-1982, June 2010 .

[8] Leon O. Chua, Introduction to nonlinear network theory, McGraw-Hill, New York, 1969.

[9] Y. Tsividis, Operation and Modelling of the Metal-oxide Semiconductor Transistor, McGraw-Hill, 1999.

[10] M. Tiebout, Low Power VCO Design in CMOS, Springer 2006. 